european journal of histochemistry

a journal of functional cytology

ISSN 1121-760X

volume 63/ supplement 1

2019

Therapeutic nanoproducts:

from biology

to innovative technology

19-20 June 2019

Aula Pocchiari

Istituto Superiore di Sanità, Rome

Co-Organized by

Istituto Superiore di Sanità (ISS)

and

Associazione Italiana di Colture Cellulari (AICC)

SCIENTIFIC COORDINATORS

Stefania Meschini

Michele Caraglia

\title{
SCIENTIFIC SECRETARIAT
}

Maria Condello

Organizing Secretariat

Anna Tobelli

Massimo Donadelli

Evelin Pellegrini

under the auspices of the University of Pavia, Italy 


\section{Published by PAGEPress, Pavia, Italy}

\section{Editorial Office:}

Via A. Cavagna Sangiuliani 5, 27100 Pavia, Italy Phone: +39.0382 .464340 - Fax: +39.0382 .34872$

E-mail: info@pagepress.org

\section{Printed quarterly by:}

Press Up s.r.l.

via E.Q. Visconti, 90, 00193 Roma, Italy

Tel. +39.0761.527351 - Fax +39.0761.527254.

\section{Annual Subscriptions}

Europe: Euro 250

All other Countries: Euro 300

Subscriptions, cancellations, business correspondence and any enquiries must be sent to PAGEPress Publications, Pavia, Italy.

Cancellations must be received before the end of September to take effect at the end of the same year.

No part of this publication may be reproduced, stored in a retrieval system or transmitted in any form or by any means (electronic, electrostatic, magnetic type, mechanical, photocopying or otherwise) without written permission by the Publishers.

Reg. Tribunale di Pavia n. 289/23.2.1984.

Recognized by the Ministero per i Beni e le Attività Culturali, Italy as a publication of high cultural value.

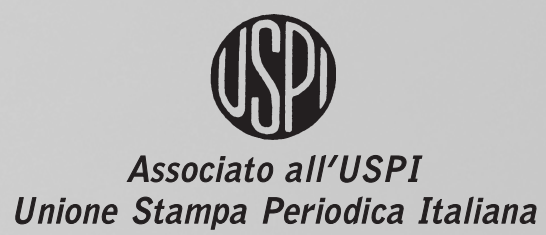

Unione Stampa Periodica Italiana

Disclaimer. Whilst every effort is made by the publishers and the editorial board to see that no inaccurate or misleading data, opinion or statement appears in this journal, they wish to make it clear that the data and opinions appearing in the articles or advertisements herein are the responsibility of the contributor or advisor concerned. Accordingly, the publisher, the editorial board and their respective employees, officers and agents accept no liability whatsoever for the consequences of any inaccurate or misleading data, opinion or statement. european journal of histochemistry

a journal of functional cytology

\section{ISSN 1121-760X \\ volume 63/ supplement 1}

\section{9}

\section{Editor in Chief}

C. Pellicciari

Dipartimento di Biologia e Biotecnologie

"Lazzaro Spallanzani" Università di Pavia

\section{Editors}

M. Biggiogera

M. Malatesta 


\section{European Journal of Histochemistry a journal of functional cytology}

The European Journal of Histochemistry was founded in 1954 by Maffo Vialli and published till 1979 under the title of Rivista di Istochimica Normale $e$ Patologica, from 1980 to 1990 as Basic and Applied Histochemistry and in 1991 as European Journal of Basic and Applied Histochemistry. It is now published under the auspices of the University of Pavia, Italy.

The European Journal of Histochemistry is the official organ of the Italian Society of Histochemistry and a member of the journal subcommittee of the International Federation of Societies for Histochemistry and Cytochemistry (IFSHC), and has been an influential cytology journal for over 60 years, publishing research articles on functional cytology and histology in animals and plants.

The Journal publishes Original Papers, Technical Reports, Reviews, Brief Reports, Letters to the Editor, Views and Comments, and Book Reviews concerning investigations by histochemical and immunohistochemical methods, and performed with the aid of light, super-resolution and electron microscopy, cytometry and imaging techniques; attention is also given to articles on newly developed or originally applied histochemical and microscopical techniques.

Coverage extends to:

- functional cell and tissue biology in animals and plants;

- cell differentiation and death;

- cell-cell interaction and molecular trafficking;

- biology of cell development and senescence;

- nerve and muscle cell biology;

- cellular basis of diseases.

\author{
Editor in Chief \\ Carlo Pellicciari (University of Pavia, Italy)
}

\section{Editors}

Marco Biggiogera (University of Pavia, Italy)

Manuela Malatesta (University of Verona, Italy)

\section{Editorial Board}

F. Cima, Padua (Italy), L. Cocco, Bologna (Italy), A.C. Croce, Pavia (Italy), G. Cutroneo, Messina (Italy), E. Falcieri, Urbino (Italy), A. Franchitto, Rome (Italy), M. Harata, Sendai (Japan), P. Hozak, Prague (Czech Republic), Z. Kmiec, Gdansk (Poland), N.M. Maraldi, Bologna (Italy) F.J. Medina, Madrid (Spain), G. Meola, Milan (Italy), S. Modina, Milan (Italy), M. Pavelka, Vienna (Austria), M.T. Perra, Cagliari (C.A. Redi, Pavia (Italy), G. Rindi, Rome (Italy), S. Shibata, Tokyo (Japan), C. Schoeffer, Vienna (Austria)

Managing Board of the Italian Society of Histochemistry for the years 2018-2021

Elisabetta Falcieri (President) University of Urbino "Carlo Bo", Italy

Antonio Franchitto (vice-President)

University of Rome "La Sapienza", Italy

Francesca Cima (Member)

University of Padua, Italy

Giuseppina Cutroneo (Member)

University of Messina, Italy

Silvia Modina (Secretary)

University of Milan, Italy

Carlo Pellicciari (past-President)

University of Pavia, Italy

\section{Editorial Staff}

Nadia Moscato, Managing Editor

Cristiana Poggi, Production Editor

Tiziano Taccini, Technical Support

2017 Impact factor: 2.217. ${ }^{\ominus} \mathrm{JCR}$ Clarivate Analytics 


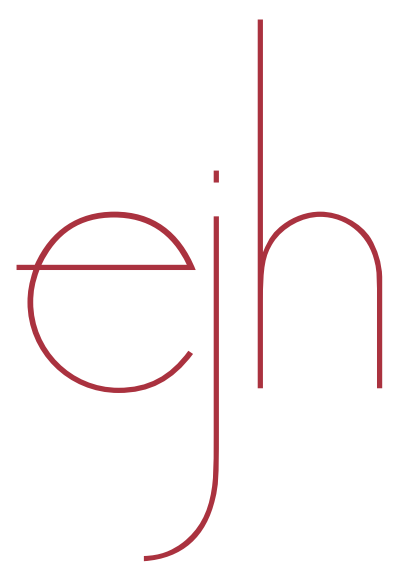

table of contents

Therapeutic nanoproducts:

from biology to innovative technology

Main Lecture

.1

Abstracts

european journal of histochemistry ISSN 1121-760X volume 63/supplement 1 2019 


\section{MAIN LECTURE}

\section{THERAPEUTIC NANOPRODUCTS: FROM BIOLOGY TO INNOVATIVE TECHNOLOGY}

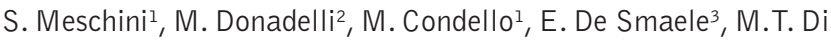
Martino ${ }^{4}$, E. Pellegrini ${ }^{1}$, C. Riganti ${ }^{5}$, K. Scotlandi ${ }^{6}$, F. Zazzeroni ${ }^{7}$, M. Caraglia ${ }^{8}$

${ }^{1}$ National Center for Drug Research and Evaluation, National Institute of Health, Rome; ' ${ }^{2}$ Department of Neurosciences, Biomedicine and Movement Sciences, Section of Biochemistry, University of Verona, Verona; ${ }^{3}$ Department of Experimental Medicine, Sapienza University of Rome, Rome; ${ }^{4}$ Department of Experimental and Clinical Medicine, Magna Graecia University, Catanzaro; ${ }^{5}$ Department of Oncology, University of Turin; ${ }^{6}$ IRCCS Istituto Ortopedico Rizzoli, Experimental Oncology Laboratory, Bologna; ${ }^{7}$ Department of Biotechnological and Applied Clinical Sciences, University of L'Aquila, L'Aquila; and ${ }^{8}$ Department of Precision Medicine, University of Campania " $L$. Vanvitelli", Naples, Italy

The main objective of every scientific progress is the improvement of wellness and human health. New strategies are necessary for safe and effective therapeutic treatments, to reduce toxicity and the side effects of conventional drugs and improve the efficiency and accuracy in the biological targeting.

This Congress aims to give an overview of all the potential therapeutic and biomedical applications and the issues that must be faced when you plan to start working with nanometric dimension materials.

The enhancement of the therapeutic efficiency of a system with these dimensions requires the acquisition of new skills, within a strategic planning that leads to the expansion and sharing of knowledge in a multidisciplinary environment. The Conference will deepen the mechanisms of interaction between new chemical-biological formulations and biophysical systems to improve the transport of drugs in normal and tumor cells. Likewise, it is important to improve knowledge and encourage the optimization of nanometric materials production methods and to discuss the regulatory aspects. Knowledge on essential questions such as characterization of nanomaterials, their hazards, the assessment and management of risks is surely improved by the numerous efforts made in biomedical research and development. The protection of health and environment needs to be increased by also improving the current laws and regulatory system.

The conference will be divided into three sessions, with the following titles:

\section{Cell Interactions of Therapeutic Vesicles; \\ 2. Advanced Biomedical Technology; \\ 3. Nanosized Innovative Approaches.}

The first session includes seven reports by eminent scientists on the therapeutic use of vesicles including liposomes, micro-vesicles and exosomes as nanocarriers. Small membranous vesicles are heterogeneous and play different biological functions, some of them can be shed from the surface of cells and have an impor- tant role in mediating intercellular communication. The bidirectional vesicular trafficking from and to the plasma membrane and subsequent redistribution in the surface of lipids contribute to intra and extracellular large vesicle formation. Tumor derived extracellular vesicles like exosomes can carry complex bioactive molecules, such as nucleic acids and proteins, which can influence the formation of metastases. The information contained in the exosomes is transmitted to adjacent tumor cells and this allows an interchange of messages between malignant cells. Moreover, these vesicles may move to distant regions of the body and modulate the microenvironment to form a pre-metastatic niche. Tumor derived extracellular vesicles can also be considered as potential biomarkers for tumor development and metastasis. Chemotherapeutic drugs can be encapsulated in liposomes, sometimes to improve their uptake and minimize side effects. Drugs are encapsulated in the liposomes so increasing the antiproliferative, cytotoxic and apoptotic effect especially in the resistant cancer cells. Deepening the knowledge of the structure, role, toxicity and fate of extracellular vesicles is important for using them as carriers to determine more specific delivery to tumor sites.

The second session includes lectures on the development of new approaches for the improvement in medical and biotechnological applications. Biomedical technology is the science that precisely controls the structure of matter at the molecular level and is widely viewed as the most significant technological frontier currently being explored in the fields of engineering and computing research. These technologies aim to exploit on the possibility of manipulating and organizing atoms or molecules in order to obtain some new medical devices that can be used to improve human health conditions. These devices can be integrated functional structures, mainly composed of mixtures of materials that exhibit peculiar properties and capabilities that are derived from the spatial-temporal organization of components, as well as from the coordination and regulation of action of individual components and their surface properties correlated to their design. The organization of these components in the medical devices may allow them to perform multistep work processes. Furthermore, many micro- and nano-devices can play a significant role in medicine even when they are not implanted and are instead used externally as wearables or as laboratory devices.

The third session will cover the study and use of nanostructured material in both inflammatory and cancer diseases. Topics concerning the use of nanocellulose, integrated microdevices, hyperthermic nanoparticles, porous silica nanovectors and the possibility of using a sensitive super-resolution technique for the imaging of dynamic biological processes will be presented. Finally, the position of nanomaterials in the framework of REACH (Registration, Evaluation, Authorisation and restriction of Chemicals) regulations will be discussed. REACH aims to improve the protection of both human health and environment through the better and earlier identification and evaluation of the intrinsic properties of chemical substances. It is the over-arching legislation, which is applicable to the manufacture, placing distribution on the market and use of substances on their own, in preparations or in articles. Until now, nanomaterials are covered only by the definition of "substances" in REACH, but there is no explicit reference to nanomaterials. Therefore, the understanding of how nanomaterials must be registered and regulated by laws is relevant for both scientists and companies.

This Congress will give an overview of all the potential applications and problems that must be solved when we start working with nanometric materials. 


\section{ABSTRACTS}

\section{MICROVESICLES AND EXOSOMES IN METABOLIC DISEASES: THEIR ROLE IN INFLAMMATION}

\author{
L. Dini ${ }^{1,2}$, S. Tacconi ${ }^{3}$, E. Panzarini ${ }^{3}$ \\ ${ }^{1}$ Dept. of Biology and Biotechnology "C. Darwin", Sapienza \\ University of Rome, Rome, Italy; ${ }^{2}$ CNR-Nanotec, Lecce, Italy; \\ ${ }^{3}$ Dept. Biological and environmental science and technology, \\ University of Salento, Lecce, Italy
}

E-mail: Iuciana.dini@uniromal.it

Multifactorial abnormalities, such as hyperglycemia, elevated triglyceride levels, low high-density lipoprotein cholesterol levels, etc., characterize metabolic diseases, whose progression and pathological features rely on the occurrence of local or systemic inflammation. Among immune cells, macrophages are critical effectors in the inflammation as diversity and plasticity are two their hallmarks: they can change the functional status from a pro-inflammatory phenotype (classical activation called MI) to an anti-inflammatory one (alternative activation called M2) in relation to the microenvironment and external stimuli. In this crosstalk, extracellular vesicles (EVs), including microvesicles ( MVs) and exosomes (EXOs), play a pivotal role as they can transport proteins and nucleic acids to adjacent cells or to distant organs. In the current study, we characterized EVs shed by in vitro differentiated (MO) THP-1 human monocytes upon hyperglycaemic stress obtained by treating MO macrophages with 15 and $30 \mathrm{mM}$ glucose for $24 \mathrm{~h}$, by using transmission and scanning electron microscopy and dynamic light scattering (DLS) analysis. Moreover, we evaluated the ability of EVs released by glucose stressed cells to interfere with macrophages polarization by culturing differentiated THP-1 cells for up to $24 \mathrm{~h}$ in the presence of MVs, EXOs and in the conditioned mediumCM without EXOs and MVs of MO glucose stressed macrophages. Genes (IL-1 $\beta$, IL-6, IL-10, IFN- $\alpha$, TNF- $\alpha$, CCL17, TLR4, NFkB) and proteins (iNOS, CD86, CD163, MHC-I, STAT6) expression was used to detect the markers of macrophage polarization. Results showed that in hyperglycemic condition, that is a strong inducer of the pro-inflammatory markers, MO macrophages are induced to an increased secretion of $E V s$, in particular of microvesicles that, in turn, enhance the Mo polarization towards a pro-inflammatory phenotype. This opens new perspectives in understanding the role of macrophages in diabetes and in the cell-cell communication based on EVs release and provide potential therapeutic targets for the treatment of inflammatory conditions during metabolic diseases.

\section{INSIGHT INTO SURFACTANT-BASED "SOFT" NANOCAR- RIERS}

M. Carafa

Department of Drug Chemistry and Technology, Sapienza University of Rome, Italy

E-mail: maria.carafa@uniromal.it

The application of nanotechnology in diagnosis and therapy is widely accepted as a potential driver of medical innovation. During the last decades, the formulation of surfactant-based "soft" nanocarriers (micelles, micro and nanoemulsions, niosomes), as a tool to improve drug delivery,brought an ever increasing interest among the scientists working in the area of drug delivery systems. In particular, niosomes ${ }^{1,2}$ are self assembled vesicular nanocarriers obtained by hydration of synthetic surfactants and appropriate amounts of cholesterol or other amphiphilic molecules. To get insight into the formulation of surfactant-based "soft" nanocarriers to develop well defined nanoformulations of therapeutic relevance an adequate characterization is of paramount importance. Determination of physico- chemical features such as particle size and morphology, surface charge and stability are indispensable to better evaluate nanocarrier proper application. Furthermore, better understanding of the extra- and intra-cellular fate of the nanocarriers in relation with biological environments in vitro and in vivo is essential to understand how dynamic interactions with biological components alter their fate and performance in vivo ${ }^{3}$. Nevertheless, the opportunities of nanotechnologies in the health sector are accompanied by challenges in the regulation of these products ${ }^{4}$. Sufficient knowledge on their quality, safety, and efficacy has to be gained to support the regulatory decision to allow the translation towards clinical applications. The highly interdisciplinary nature of this field, from basic chemistry and physics to clinical evaluations, makes researches in developing nanomedicines very fruitful. This means there are still many open challenges that need to be overcome and there are still opportunities for academic and industrial scientists to make a decisive impact.

\section{References}

1. Marianecci C, et al. Adv Colloid Interface Sci 2014;205:187-206.

2. Moghassemi S, Hadjizadeh A. J Contr Rel 2014;185:22-36.

3. Seo SJ, et al. Nano Today 2017;14:84-99.

4. Bremer-Hoffmann S, et al. J Interdisc Nanomedicine 2018;3:4-15.

\section{MESENCHYMAL STROMAL FREEZE-DRIED EXTRACEL- LULAR VESICLES FOR NANOMEDICINE: FROM GMP-COMPLIANT PRODUCTION TO NEXT GENERATION DRUG DELIVERY SYSTEM}

\author{
S. Perteghella, E. Bari, M.L. Torre
}

Department of Drug Sciences, University of Pavia, Italy

E-mail: sara.perteghella@unipv.it

Mesenchymal Stem Cells (MSCs)-secretome, composed of soluble proteins and extracellular vesicles (EVs), represents a new therapeutic approach for chronic and acute diseases in place of parental cells. A perspective view is here provided about the state-of-art and the missing steps required for the secretome "pharmaceuticalization" into a high quality, safe and effective medicinal product, as well as for optimal formulation. A scalable and GMP-compliant production process is the first requirement and it is now established that the ultrafiltration is the only one that fit. Stability and shelf-life are the second issue: freeze-drying is the technological process widely used in pharmaceutical field, which is best suited to both protein and EVs. We combined ultrafiltration and lyophilisation techniques obtaining a ready-to-use powder, lyo-secretome ${ }^{1-3}$. The process did not affect EV integrity and morphology, and allowed to obtain a safe and effective product, which can be considered as a new Active Pharmaceutical Ingredient (API). Efficacy of lyo-secretome was proven against oxidative stress-induced damages ${ }^{2}$, as immunomodulant agent ${ }^{3}$ and in a wound healing murine model. Furthermore, proteomic characterization of lyo-secretome revealed that it contains alphal-antitripsin (AAT) with in vitro anti-elastase activity, paving the way for the use in lung degeneration AAT deficiency. At the same time, characteristics of EVs make them ideal candidates as drug delivery systems (DDS), due to their high stability in bloodstream and low immunogenicity. Moreover, the innate homing ability to injured site, make EVs suitable smart DDS to avoid off side effects, and enhance the specific uptake by target cells. In this context, EVs can also be used as "carrier in carrier" device constituted by EVs containing nanosystems loaded of drugs $^{4}$. These results demonstrate that MSC-secretome can be used both as API and DDS; although human and veterinary clinical trials are still few, we are confident that many will start in the near future.

\section{References}

1. Perteghella et al. PCT/IB2017/056591;2016.

2. Bari E, et al. Cells 2018;7. pii:E190. 
3. Bari $E$, at al. Nanomedicine $2019 ; 14: 753-65$.

4. Perteghella S, et al. Int J Pharm 2017;520:86-97.

\section{NANOMEDICINE IN CANCER: TOWARD OVERCOMING CHEMORESISTANCE}

M. Caraglial, C. Leonetti ${ }^{2}$, C. Riganti ${ }^{3}$, V. Campani ${ }^{4}$ S. Zappavigna', M. Porru², J. Kopecka ${ }^{3}$, M. Abate ${ }^{1}$, G. De Rosa ${ }^{1}$

${ }^{1}$ Department of Biochemistry, Biophysics and General Pathology, University of Naples "Vanvitelli", Naples; 'UOSD SAFU, IRCCS Regina Elena National Cancer Institute, Rome; ${ }^{3}$ Department of Oncology, University of Turin, Turin; and ${ }^{1}$ Department of Pharmacy, Federico II University of Naples, Naples, Italy

\section{E-mail:gderosa@unina.it}

Intrinsic and acquired drug resistance (multidrug resistance or MDR) is a major challenge in treating cancer patients. One of the most frequent cause of chemoresistance is the elevated expression levels of drug efflux pumps, such as P-glycoprotein (Pgp) belonging to the ATP-binding cassette (ABC) superfamily. Nanomedicine represents a promising tool to overcome MDR at different levels. First of all, nanovectors have been proposed to deliver $A B C$ inhibitors co-administered or co-encapsulated with chemotherapeutics. Molecules with a well-established activity of ABC-inhibitors, such as verapamil, have showed a systemic toxicity, while alternative strategies to inhibit $A B C$ expression are emerging e.g., with the bisphosphonates as zoledronic acids (ZOL). Recently, we demonstrated that the use of nanovectors, such as liposomes and self-assembling nanoparticles (SANPs) can be used to deliver $\mathrm{ZOL}$ in tumors ${ }^{1,2}$. We also observed that ZOL, only by using nanovectors, reduces the expression of Pgp in MDR cancer cells 3,4 , thus restoring the sensitivity of MDR tumors to chemotherapeutics such as doxorubicin or carboplatin ${ }^{4}$. More recently, chitosan-based nanoparticles have been developed to co-encapsulate ZOL and doxorubicin for combined therapy against MDR tumors ${ }^{5}$. In this case, also a synergistic inhibition activity was observed when combining ZOL and doxorubicin on MDR cells ${ }^{5}$. A second level in which the nanomedicine can be used to overcome the MDR is the delivery of nucleic acids such as small interfering RNA (siRNA) or microRNA (miRNA). Indeed, siRNAs can be used to inhibit the expression of a protein involved in MDR, while miRNAs are important modulators or protein expression and their misexpression has been correlated to the occurrence of MDR. Thus, polymeric micelles have been developed for the co-delivery of an anti-survivin siRNA and paclitaxel for the reversal of drug resistance in tumors 6 . On the other hand, SANPs encapsulating the miR603 have been proposed to restore sensitivity to temozolomide in glioblastoma cells (unpublished data). Finally, a third level of attention should be paid on the biomaterials used when using nanovectors in MDR tumors. The use of liposomes can overcome the resistance to doxorubicin in cells overexpressing Pgp. In addition, the use of suitable biomaterials, and in particular the inclusion of PEGylated lipid into the formulation, can be particularly useful to inhibit activity of the efflux pumps.

\section{References}

1.Salzano et al. 2011.

2. Marra et al. 2012

3. Kopecka et al. Oncotarget -2015

4. Kopecka et al. Oncotarget -2016

5 Giarra et al. Pharmaceutics 2018

6.Salzano G, et al. Mol Cancer Ther. 2015

7. Kopecka et al. Nanomedicine 2014

\section{HACKING PANCREATIC CANCER DRUG RESISTANCE WITH NOVEL NO-GEMCITABINE PRODRUGS ENCAPSU- LATED IN LIPOSOMES}

F. Masetto1, K. Chegaev², E. Gazzano3, N. Mullappily', B. Rolando ${ }^{2}$, R. Fruttero ${ }^{2}$, S. Arpicco ${ }^{2}$, C. Riganti ${ }^{3}$, M. Donadelli ${ }^{1}$

${ }^{1}$ Department of Neurosciences, Biomedicine and Movement Sciences, University of Verona; ${ }^{2}$ Department of Drug Science and Technology, University of Turin; 'Department of Oncology, University of Turin, Italy

\section{E-mail: massimo.donadelli@univr.it}

Since the late 1990 's, gemcitabine (GEM) has been available as the standard of care regimen for advanced pancreatic ductal adenocarcinoma (PDAC). However, the resistance to chemotherapy treatment is the main issue against a successful anti-cancer therapy. ${ }^{1}$ Nitric oxide (NO) is a small free radical molecule involved in many physiologic and pathologic pathways in human cells. It plays also a key role in tumor biology and at micromolar concentrations it displays antitumor effects. ${ }^{2,3}$ In this study, we synthesized a library of seven NO-releasing gemcitabine conjugates (NO-GEMs) in order to improve the effectiveness of GEM in PDAC cell lines. We used as a cellular model of PDAC Pancl and MiaPaca2 cell lines, which were previously demonstrated to be more resistant or sensitive to the GEM treatment, respectively. Among the new NO-GEM prodrugs, we identified GCB2 as the most effective compound in inhibiting cancer cell growth. To improve its uptake and minimize side effects we encapsulated it in liposomes (LIPO-GCB2), increasing its antiproliferative and apoptotic effect especially in GEM-resistant Pancl cells, as compared to standard GEM. We observed that the multidrug resistant (MDR) family member MRP5, which is involved in the cellular export of GEM, is more expressed in GEM-resistant than in GEM-sensitive PDAC cell lines. Notably, LIP0-GCB2 inhibited MRP5 activity by nitrosylation, favoring the intracellular accumulation of GEM and triggering apoptotic cell death in GEMresistant PDAC cells. Our results support the development of a new anti-tumoral strategy to efficiently affect GEM-resistant PDAC cells based on the usage of NO-releasing GEM encapsulated in liposomes.

\section{References}

1. Binenbaum $\mathrm{Y}, \mathrm{Na}^{\prime} \mathrm{Ara} \mathrm{S}$, Gil Z. Drug Resist Updat 2015;23:55-68.

2. Huerta S, Chilka S, Bonavida B. Int J Oncol 2008;33(5):909-927.

3. Riganti C, et al. Cancer Res 2005;15;65:516-25.

\section{LIPOSOMAL CARRIER AS POSSIBLE THERAPEUTIC VESICLES: MORPHO-FUNCTIONAL INVESTIGATION}

\section{Battistelli}

Department of Biomolecular Sciences (DiSB), University of Urbino Carlo Bo, Italy

\section{E-mail: michela.battistelli@uniurb.it}

In the last decades, deficiency of macro- and micronutrients was considered as a serious problem associated with the increase in the human population. Micronutrient malnutrition causes blindness and anemia (even death) in more than half of the world's population, especially among women of reproductive age, pregnant and lactating women, and preschool children. In particular, iron deficiency is one of the most frequent health problems, affecting approximately one third of the entire population. Its request may not be satisfied with a regular diet, due to a low iron bioavailability and to dietary factors that limit its absorption. Iron supplementation is considered a global economic and effective strategy for prevention and control of anemia, in particular, during pregnancy ${ }^{1}$. Therefore, there is the need to develop new experimental protocols to improve its bioavailability. Liposomes ${ }^{2}$ are vesicular structures with double-layer lipid membrane, which are proved particularly interesting in the biomedical field as a delivery system of pharmaceutically active substances ${ }^{3}$. In this study, ultrastructural analyses have been carried out to verify if 
liposome dehydration process induces structural alterations of lipid membranes, which could compromise carrier integrity and function. Morphological integrity of Biofer and Lifervit (two new commercial iron carriers), both in liquid form and in dried powder, has been investigated by means of transmission electron microscopy ${ }^{4}$. Moreover, liposome interaction with U937 cell line, a well-known phagocytosis human model, has been studied. Both compounds revealed a good stability and the ability to penetrate into cells, interacting with cytoplasmic organelles without inducing, at least apparently, any ultrastructural damage and toxicity. Therefore, Biofer and Lifervit do not cause cellular damage, and for that they can be considered potential candidates in iron vehiculation. Further studies are in progress to evaluate their interaction with intestinal cell models.

\section{References}

1. Kamau MW, et al. BMC Public Health 2018;18:580.

2. Tamjidi F, et al. Innov Food Sci Emerg Technol 2014;26:366-74.

3. Keshari SA, et al. Pharmanest 2014;5:2019-33.

4. Battistelli M, et al. Microsc Res Tech 2018;81:1295-1300.

\section{IMMUNOTOXICITY ASPECTS IN THE DEVELOPMENT AND REGULATORY EVALUATION OF NANODRUGS}

\author{
G. Di Felice, B. Barletta, C. Butteroni, S. Corinti
}

National Center for Drug Research and Evaluation, Istituto Superiore di Sanità, Rome, Italy

E-mail: gabriella.difelice@iss.it

Innovative nanotechnology-based medicinal products are increasingly developed for both therapeutic and diagnostic uses. The challenge for regulatory agencies is to ensure the proper evaluation of the quality, safety, and effectiveness of nanomedicines undergoing clinical development and market authorization. For a safe translation into clinical applications, proper regulatory pathways and standardised test methods must be available. The primary function of immune system is to protect the host from foreign invaders, by integrating the actions of its innate and adaptive compartments. Upon the intentional delivery of nanodrugs or diagnostic/imaging systems, they interact with immune cells that are abundant in the tissues close to the entry sites as well as in the bloodstream. Because of nanodrugs interaction with the immune system, the assessment of their impact in terms of immunotoxicity, and of their potential to modulate its function (suppression, stimulation, and hypersensitivity) deserves adequate attention in the regulatory risk-benefit assessment ${ }^{1}$. Until now, no specific regulatory documents have been developed to assess the immunotoxicity of nanodrugs, which are currently evaluated on the basis of guidelines, such as the ICHS8 Note for Guidance on Immunotoxicity Studies for Human Pharmaceuticals ${ }^{2}$ in force for conventional medicinal products. The crucial question is whether the set of first- and second-level tests prescribed by these guidelines is capable of adequately evaluating the immunotoxic profile of complex and broadly heterogeneous nanodrugs that result from the combined effects of the active pharmaceutical component, the nanocarrier, any potential coating, and the final formulation ${ }^{3}$. To address this question, we developed a panel of in vitro assays on immunocompetent cell lines to evaluate cytotoxicity, functional activation, production of effector molecules (Nitric Oxide, NO) and proinflammatory mediators (IL-6 and TNF $\alpha$ cytokines). Two cell lines of murine origin, RAW264.7 and MH-S, have been selected being representative of different exposure conditions and entry site delivery, since they belong to the monocyte-macrophage lineage from the systemic circulation and the lung alveolar district, respectively. The results obtained in these simple and reproducible systems support their predictivity and potential application both to the safe-by-design development of nanodrugs and to the implementation of nano-specific regulatory guidelines.
1. Dobrovolskaia MA, et al. Toxicol Appl Pharm 2016:299:78-89.

2. http://www.ema.europa.eu/docs/en_GB/document_library/ Scientific_guideline/2009/09/WC500002851.pdf

3. Giannakou C, et al. Int J Nanomedicine 2016;11:2935-52.

\section{NANOSTRUCTURED MATERIALS AND DEVICES}

M. Grigioni ${ }^{1}$, B. De Berardis ${ }^{1}$, G. D Avenio $^{1}$, C. Daniele ${ }^{1}$, G. Bozzuto ${ }^{2}$, M. Condello ${ }^{2}$, S. Meschini ${ }^{2}$, A. Molinari' ${ }^{2}$ R. Pecci ${ }^{1}$, R. Bedini ${ }^{1}$, F. Superti ${ }^{1}$

${ }^{1}$ National Center for Technological Innovation in Public Health; ${ }^{2}$ National Center for Drug Research and Evaluation, Istituto Superiore di Sanità, Rome, Italy

E-mail: mauro.grigioni@iss.it

The increasing diffusion of nanomaterials ( NM) in the domain of medical devices (MD) is due to the peculiar characteristics of such materials (e.g., the tunability of their physico-chemical properties as a function of their size), which allow to obtain a high biocompatibility and, at the same time, to exploit their high surface/volume ratio. Clearly, the associated risks must carefully be considered, besides their advantages. The need for regulatory oversight of NM-containing DM is reflected by the new MD Regulation: MD incorporating or consisting of NM are in class III, unless the NM is encapsulated or bound in such a manner that it cannot be released into the patient's or user's body. In order to identify possible mechanisms of cell-NM interaction, the cell damage potential of some relevant NM ( $\mathrm{ZnO})$, already used in the fabrication of MD, was assessed by means of different techniques (MTT, electric cell-substrate impedance sensing ECIS, SEM , TEM ,...). Moreover, we selected a nanostructured wound dressing product containing Ag NM already used in the fabrication of MD. The cell damage potential of these NMs was assessed, again using different techniques. The guidance for dealing with NM-associated risks in the European regulatory framework was investigated. Standard testing on the final product must be considered, besides the typical verification activities of the Notified Bodies (third part entities for conformity assessment). The standard cytotoxicity assays showed a good agreement with ECIS, which gives a continuous real-time measurement of cell viability, and provides valuable information on cell damage mechanisms. The results were very sensitive to NM concentration, showing the necessity of defining clear thresholds for each NM and contact type. In particular, it is generally agreed that the ISO 10993 series must be revised in view of the specificity of NM. Methodological suggestions have been provided in ${ }^{1}$, stating that "it is likely that the various assays as described in the ISO 10993 series are not always appropriate as such in the testing of nanomaterials." Notably, the accuracy of traditional cytotoxicity tests may fail, due to the interaction between NMs and assay materials. In our experience, a satisfactory agreement was found between traditional and innovative (ECIS) tests. A cautionary approach about nanostructured MDs is suggested by the new EU regulatory framework, in view of the still incomplete knowledge about them.

\section{Reference}

1. ISO/TR 10993-22:2017 Biological evaluation of medical devices Part 22: Guidance on nanomaterials 


\section{POLYMERIC NANOPARTICLES AS VERSATILE DRUG DELIVERY SYSTEMS}

\author{
D. Cosco \\ Department of Health Sciences, University of Catanzaro "Magna \\ Græcia", Campus Universitario "S. Venuta", Catanzaro, Italy \\ E-mail: donatocosco@unicz.it
}

Polymeric nanoparticles have been extensively investigated as drug delivery systems due to their technological features. They can be classified into two types based on their morphology and these are capsules, which are systems made up of a drug-containing core surrounded by a polymeric shell, and spheres, usually characterized by a matrix in which the active compounds are contained or adsorbed. In particular, aqueous-core polymeric nanocapsules are used to modify the biopharmaceutical properties of water-soluble drugs, thus increasing their plasmatic halflife and pharmacological efficacy besides decreasing their side effects. For example, novel aqueous-core polylactic acid (PLA) nanocapsules able to retain hydrophilic active compounds have been developed and their physico-chemical properties evaluated as a function of the components used. The peculiar structure of these nanosystems favoured a massive encapsulation of antitumor drugs increasing their in vitro and in vivo anticancer efficacy with respect to their free forms ${ }^{1}$. The rigid shell of PLA nanocapsules allows the efficient entrapment and physical retention of genetic material. For instance, plasmids and siRNAs have been efficiently encapsulated and protected from degradation, and the resulting nanoplexes have evidenced good cell transfection features $^{2}$. The polymeric nanosystems also permitted the efficient entrapment of meglumine antimoniate (glucantime), increasing its accumulation in macrophages and thus its pharmacological activity against Leishmania tropica ${ }^{3}$. Another interesting biopolymer used to obtain nanosystems is zein, a natural hydrophobic protein belonging to alcohol-soluble prolamine-rich compounds abundantly contained in corn. Zein nanoparticles with a mean diameter of 100-150 nm have been developed by using various surfactants. These systems are characterized by a great degree of stability and are able to retain various active compounds ${ }^{4}$. The results evidence the potential clinical application of these polymeric formulations as novel drug delivery systems.

\author{
References \\ 1. Cosco D, et al. Eur J Pharm Biopharm 2015;89:30-39. \\ 2. RM2013A000635. \\ 3. RM2014A000252, PCT W02015/177820. \\ 4. Gagliardi A, et al. Int J Nanomedicine 2018;13:601-14.
}

\section{MESOPOROUS SILICA-BASED NANOSYSTEMS AS A VEHICLE FOR TARGETED DELIVERY OF THE ANTINEO- PLASTIC DRUG BORTEZOMIB}

\author{
A. Leggio $^{1,2}$ \\ Rende, Italy \\ E-mail: antonella.leggio@unical.it
}

${ }^{1}$ NanoSiliCal Devices S.r.l., University of Calabria, 87036 Arcavacata di Rende, Italy; ${ }^{2}$ Department of Pharmacy, Health and Nutritional Sciences, University of Calabria, Arcavacata di

Traditional cancer therapies are often limited by the lack of selectivity to cancer cells as they frequently display unwanted severe toxicities on the healthy ones. Pharmacological properties of conventional anticancer drugs can be improved through the use of drug delivery systems (DDS) composed of biocompatible nanomaterials that can deliver therapeutic agents selectively to cancer cells while sparing normal tissues and minimizing side effects ${ }^{1}$. The intrinsic properties of mesoporous silica nanoparticles (MSN) make them ideal delivery platforms ${ }^{2}$. Their structural and chemical features as high surface area, tunable size, and easy surface modification, permit to design versatile nanosystems able to encapsulate a variety of therapeutic agents and to host targeting molecules able to interact selectively with specific membrane receptors overexpressed in tumor cells. A MSN-based device, FOL-MSN-BTZ, for $\mathrm{pH}$-sensitive release of the antineoplastic drug bortezomib (BTZ) in cancer cells over-expressing folate receptor (FR) has been developed. It consists of a nanometric honeycomb-like silica frame that bears on the external surface folic acid (FOL) as targeting function (folate receptor specific ligand) and in the inner pores the antineoplastic drug BTZ linked by means of a pH-sensitive bond. The boronic acid group of BTZ interacts with diol functionalities on the MSNs pore surface by forming cyclic boronic esters that are quite stable and are hydrolysed only in acidic environments, such as those of lysosomes and endosomes once target tumour cells are reached; the lower $\mathrm{pH}$ encountered therein triggers the drug release. FOLMSN-BTZ was tested on cancer cells overexpressing the folate receptor ( $F R+$ cells) and on FR normal cells, in order to investigate MSNs behavior. FOL-MSN-BTZ was able to induce death exclusively in FR+ tumor cells, while free BTZ resulted toxic for all cell lines tested, including FR normal cells.

\author{
References \\ 1. Peer D, et al. Nat Nanotechnol 2007;2:751-60. \\ 2. Argyo C, et al. Chem Mater 2014;26:435-51.
}

\section{MESENCHYMAL STEM CELLS AS A VEHICLE FOR MUL- TIMODAL NANOPARTICLES}

E. Martella ${ }^{1}$, B. Dozza², C. Bellotti ${ }^{3}$, S. Lenna 3 , M. Columbaro3 A. Guerrini ${ }^{1}$, C. Ferroni ${ }^{1}$, G. Sotgiu ${ }^{1}$, L. Cevolani ${ }^{3}$, E. Lucarelli ${ }^{3}$, G. Varchi ${ }^{1}$, D.M. Donati ${ }^{2,3}$, S. Duchi ${ }^{1}$

${ }^{1}$ ISOF-CNR, Bologna; 2Dept Biomedical and Neuromotor Sciences, University of Bologna; ${ }^{3}$ IRCCS Istituto Ortopedico Rizzoli, Bologna, Italy

\section{E-mail: elisa.martella@isof.cnr.it}

In the past decade, the use of human Mesenchymal Stem Cells (hMSC) as cell based drug delivery vectors to treat cancer, has significantly developed. hMSC display the capacity to internalize and retain drugs and nanoformulations ${ }^{1}$, and they can infiltrate the tumor stroma via cytokines-driven clues released from the tumor $^{2}$. Osteosarcoma (OS) is the most common bone cancer diagnosed in children and adolescents. The development of chemoresistance and lung metastasis limits the success rate to a worldwide 5-years patients survival rate of $70 \%$. Therefore, OS therapy might be moving toward nanotechnology-based drug delivery systems (DDS) to reduce the cytotoxicity of antineoplastic drugs and improve their pharmacokinetics ${ }^{3}$. As well, photodynamic therapy (PDT) represents an approach to kill OS cells circumventing chemoresistance $e^{4,5}$. The aim of this study is to engineer hMSC carrying DDS (hMSC-DDS) for OS treatment, using a combination of PDT (Chlorin e6 as photosensitizer) and chemotherapy (Paclitaxel-PTX, as antineoplastic) and test its efficacy in a 3D-spheroid OS model. DDS were prepared from high molecular weight and hydrosoluble keratin functionalized with Ce6 and loaded with PTX to obtain PTX-Ce6@ker nanoparticles (KNPS) ${ }^{6}$. Our results show that hMSC efficiently internalize KNPs after $24 \mathrm{~h}$ and retain them intracellularly for several days in culture. The migratory capacity is not impaired by PTX internalization, and in the 3D model, the cell death induced by PTX-cell cycle blockage and light irradiation is $95 \%$. Our data corroborate the use of MSCs-DDS as an high effective treatment in a 3D tumor model, and set the basis for the preclinical translation. If proved to be effective in vivo, this approach 
could revolutionize OS pharmacological treatment in affected patients.

References

1. Duchi S, et al Bioconjug Chem 2014;12:649-55.

2. Studeny M, et al Can Res 2012;62:3603-8.

3. Patra JK, J Nanobiotechnology 2018; 16(1):7.

4. de Miguel GC, et al. Photodiagnosis Photodyn Ther 2018;21:79-85.

5. Duchi S, et al. J Control Release 2013;168:225-37.

6. Martella E, et al. Int J Mol Sci 2018,19:3670.

\section{VITVO: MIMICKING IN VIVO COMPLEXITY BY THE INNOVATIVE 3D MODEL}

O. Candini ${ }^{1}$, G. Grisendi ${ }^{1}$, E.M. Foppiani ${ }^{1}$, M. Brogli ${ }^{1}$, B. Aramini ${ }^{2}$, V. Masciale ${ }^{3}$, C. Spano ${ }^{1}$, T. Petrachi ${ }^{4}$, E. Veronesi ${ }^{4}$, P. Conte ${ }^{5}$, G. Mari ${ }^{1}$, M. Dominici ${ }^{1,3}$

${ }^{1}$ Rigenerand srl, Medolla, Modena; 'Division of Chest Surgery, ${ }^{3}$ Division of Oncology, Department of Medical and Surgical Sciences for Children \& Adults, University-Hospital of Modena and Reggio Emilia, Modena, Italy; ${ }^{4}$ Technopole of Mirandola, Mirandola, Modena, Italy; ${ }^{5}$ Department of Surgery, Oncology and Gastroenterology, University of Padua, Istituto Oncologico Veneto IRCCS, Padua, Italy

Three-dimensional (3D) cell culture models can provide physiologically relevant information compared with the traditional twodimensional (2D) cell cultures, generating interest in drug discovery to predict response to drugs and putative compounds, also utilizing a personalized approach ${ }^{1,2}$. Several 3D cell culture technologies have been developed to better mimic in vivo complexity, however, simple and reproducible systems able to rapidly rebuild tissue ex vivo in 3D are needed to overcome some limitations and offer novel possibilities ${ }^{3-5}$. We developed an isolated "smartphone-like" 3D cell culture bioreactor that can be loaded with tumor and/or normal cells in combination, which can be monitored using a variety of read-outs. This biocompatible device, called VITVO, sustained a 3D growth of several tumors, such as pancreatic and breast adenocarcinoma, sarcoma and glioblastoma. Cells repopulated the 3D matrix inner core, which is completely separated from the outer space by two gas-permeable membranes and can be monitored in real-time using both fluorescence microscopy and luminometry, even after transportation. The use of VITVO in a variety of assays focusing on oncology is reported and the efficacy of chemotherapy, biologics and cellbased anti-cancer agents was tested by comparing VITVO with an in vivo preclinical xeno-transplant model. Notably, the system was challenged using primary tumor cells harvested from lung cancer patients as an innovative predictive functional assay for cancer responsiveness to a checkpoint inhibitor, such as nivolumab. The novel features as well as the flexibility offered by this platform within the different approaches suggest the potential of VITVO as an innovative 3D in vitro model for pre-clinical testing with a possible relevant impact in other areas beside oncology field.

\section{References}

1. Antoni D, et al. Int J Mol Sci 2015;16:5517-27.

2. Fitzgerald K A, et al. J Control Release 2015;215:39-54.

3. Shin, C S, et al. Mol Pharm 2013;10:2167-75.

4. Fischbach C, et al. Nat Methods 2007;4:855-60.

5. Horning J L, et al. Mol Pharm 2008;5:849-62.
SEC MEETS TRPS: AUTOMATED ISOLATION AND HIGHPRECISION ANALYSIS OF EXTRACELLULAR VESICLES

C. Roesch ${ }^{1}$, A.K. Pal' ${ }^{2}$ S. Harrel ${ }^{3}$, R. Vogel ${ }^{2}$, M. Broom²

${ }^{1} I z o n$ Science Europe Ltd, Lyon, France; ${ }^{2} I z o n$ Science Ltd, Medford, MA, USA; 3 Izon Science Ltd, Christchurch, New Zealand

\section{E-mail:camille.roesch@izon.com}

Extracellular Vesicles (EVs) derived from biological fluids possess extensive heterogeneity with regards to size, number, membrane composition and cargo. Tremendous research interest exists towards development and use of EV fraction of biofluids as rich sources of diagnostic and prognostic biomarkers. High precision fractionation of the nano-biological content of biofluids can dramatically reduce background, increase purity, and inform on the biology of the biomarkers and therapeutic biomolecules. Size exclusion chromatography (SEC) is the most standardizable technique, already widely used for the purification of $E V S$ from biofluids. ${ }^{1}$ Significant improvement to the use of SEC is possible through automation and precision. Here, we developed a range of SEC columns of various sizes, with 2 resin types, separating down to $35 \mathrm{~nm}$ or $70 \mathrm{~nm}$. We also developed a low-cost prototype automatic fraction collector (AFC) that adds high precision, improves repeatability, speeds up workflow. RFID tags are proposed to ensure high quality of data capture and transfer. Moreover, Tunable Resistive Pulse Sensing technology was used for accurate, high-resolution particle analysis (size, size range, concentration, and electrophoretic mobility) and normalization ${ }^{2}$. SEC columns provide a convenient, reproducible, and highly effective means of eliminating $>99 \%$ of non-vesicular protein from biological fluid samples, and separating exosomal and nonexosomal volumes for further downstream analysis. $35 \mathrm{~nm}$ pore sized SEC gel leads to increased resolution, higher yield and one fraction earlier elution of EVs from plasma compared to the 70 $\mathrm{nm}$ pore size. Use of AFC allowed precise massbased measurements and tunability within $30 \mu \mathrm{L}$ of volume exiting the column. Most importantly, due to the additional functionality provided by $A F C$, the EV field needs to revisit the way that fraction numbers, post-SEC are used. That will be replaced with a more logical framework, wherein the void volume is measured and disposed of, and precise volumes are used instead of the somewhat arbitrary fraction numbers. Thus, the qEV-AFC platform allows for QA, high-precision $E V$ volume collection and minimizes samples processing related reproducibility issues for clinical studies.

\section{References}

1. Boing AN, et al. J Extracell Vesicles 2014;3:23430.

2. Pedersen S. ISEV 2015 meeting, Washington DC.

\section{NANOTECH 5.0: FROM BIOLOGY TO VIRTUAL REALITY, FAST FORWARD}

\author{
M. Mariani ${ }^{1}$, L. Clario², V. Brambilla ${ }^{1}$
}

${ }^{1}$ Media System Lab S.r.l., Macherio (MB), Italy; ${ }^{2}$ Nanolive SA, Ecublens, Switzerland.

\section{E-mail:matteo.mariani@m-s.it}

By combining holography with tomography, Nanolive technology is an example of emerging smart nanoscopic technique. The 3D Cell Explorer microscope guarantees three-dimensional high spatio-temporal resolution imaging of transparent unlabelled specimens, with the advantage over fluorescence techniques of not requiring sample labelling, thus reducing potential damages to living cells. Thanks to the absence of photo-toxicity and the incubated controlled environment, cells can be indeed cultured and monitored in continuous time-lapse for days. Moreover, based on the high sensitivity in Refractive Index measurements, the system enables for high resolution visualization of intracellular compartments (i.e., mitochondria, lipid droplets, lysosomes). Offering an optimal combination between sample health, spatiotemporal 
resolution and signal-to-noise ratio, holotomography represents nowadays one of the best solutions for long term live cell imaging applications.

\section{NANOCELLULOSE AND ITS COMPOSITES IN BIOMEDI- CAL DEVICES}

C.A. Maestri ${ }^{1}$, N. Ferrari ${ }^{1}$, S. Meschini ${ }^{2}$, E. Pellegrini ${ }^{2}$ M. Condello², M. Scarpa ${ }^{1}$

${ }^{1}$ Department of Physics, University of Trento, Povo-Trento; and ${ }^{2}$ National Center for Drug Research and Evaluation, Istituto Superiore di Sanità, Rome, Italy

E-mail:marina.scarpa@unitn.it

Nanosized cellulose (NC) is a material of natural origin obtained by an eco-friendly procedure, in the form of fibrils (NCF) or crystallites (CNC). In particular, CNC prepared by carboxylation and ultrasonic dispersion according to Saito et al. ${ }^{1}$, are rod-shaped, rigid and regular nanostructures with thickness 3-5 nm and 250$500 \mathrm{~nm}$ length ${ }^{2,3}$. The high surface area together with the strong hydrogen bonding and cation coordination capacity, favour the interaction of CNCs with the surrounding species. Accordingly, CNCs are easily dispersed in water, while undergo a layer-bylayer assembly when dried and entangle in soft hydrogels by a cation-driven process. CNC-derived materials show unique features in term of structure and functional properties and are suitable for the fabrication of medical and life science devices. However, the nanotoxicity of CNC as component of biomedical devices is still an open question. In this presentation we will report about the fabrication of $\mathrm{CNC}$ hydrogels and their incorporation in nanocomposite materials. The material properties such as microstructure, mechanical performances, and in vitro toxicity are also presented.

\section{References}

1. Isogai $A$, et al. Nanoscale 2011;3:71-85.

2. Maestri CA, et al. Mater Chem B 2017;5:8096-104.

3. Maestri CA, et al. Sci Rep 2017;7:11129.

\section{NANOCARRIERS FOR NEUROMUSCULAR DISEASES}

M. Costanzo ${ }^{1}$, F. Carton ${ }^{1,2}$, V. Guglielmi ${ }^{1}$, L. Calderan ${ }^{1}$, M. Repellin'1,2*, G. Lollo ${ }^{2}$, I. Andreana ${ }^{3}$, S. Arpicco ${ }^{3}$, B. Stella ${ }^{3}$, M. Malatesta ${ }^{1}$

${ }^{1}$ Dept. of Neurosciences, Biomedicine and Movement Sciences, University of Verona, Italy; ${ }^{2}$ University of Lyon, Université Claude Bernard Lyon 1, CNRS, LAGEPP UMR 5007, France; ${ }^{3}$ Dept. of Drug Science \& Technology, University of Turin, Italy

E-mail: manuela.malatesta@univr.it

Myotonic dystrophy type 1 and 2 (DMI and DM2) are genetic disorders caused by CTG and CCTG expanded repeats, respectively. The transcribed expanded RNAs accumulate in nuclear foci where splicing factors are sequestered, thus causing a general splicing deregulation in multiple tissues. In both DMs, pathological traits are muscle weakness, dystrophy/atrophy and myotonia, with disabling effects and possibly premature death. Currently, no therapy is available for DMs. Different therapeutic molecules have been successfully tested in experimental models to target the expanded RNAs; however, most of the delivery or toxicity problems remain unsolved. Our research aims at designing a novel therapeutic strategy using biocompatible nanoparticles (NPs) to administer drugs or oligonucleotides able to counteract RNA toxicity in DMs, with special reference to skeletal muscle. Drug-loaded NPs may improve therapeutic efficacy by protecting the encapsulated molecules from enzymatic degradation and ensuring their delivery and sustained release inside the cell. Among the different NPs we tested, poly(lactide-co-glycol- ide) (PLGA) NPs and hyaluronic acid (HA)-based NPs proved to be highly biocompatible for cultured murine and human myoblasts and myotubes ${ }^{1,2}$. We elucidated the mechanisms of their uptake, distribution and degradation by fluorescence and transmission electron microscopy. We also investigated NPs distribution in explanted mouse skeletal muscles maintained under fluid dynamic conditions ${ }^{3}$. Both PLGA and HA NPs proved to efficiently load pentamidine, one of the promising therapeutic agents, and preliminary results on cultured myoblasts from DM 1 patients demonstrated that pentamidine delivered by PLGA NPS reduced the pathological accumulation of nuclear foci with limited cytotoxic effects.

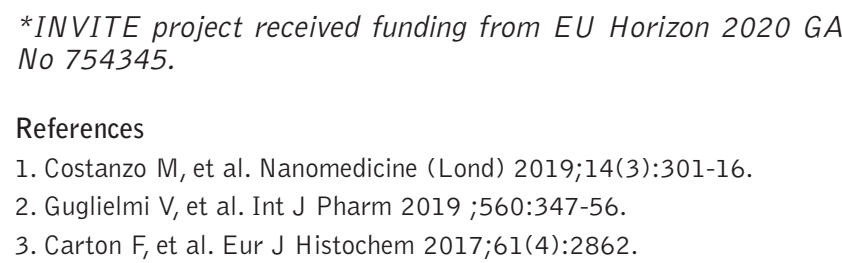

* INVITE project received funding from EU Horizon 2020 GA No 754345.

References

1. Costanzo M, et al. Nanomedicine (Lond) 2019;14(3):301-16.

2. Guglielmi V, et al. Int J Pharm $2019 ; 560: 347-56$.

3. Carton F, et al. Eur J Histochem 2017;61(4):2862.

\section{HYPERTHERMIC NANOPARTICLES TO TRIGGER LIPOLYSIS}

L. Calderan $^{1}$, F. Vurro ${ }^{1}$, S. Mannucci ${ }^{1}$, F. Boschi ${ }^{2}$, S. Tambalo ${ }^{3}$, P. Lievens ${ }^{1}$ D. Prosperi ${ }^{4}$, M. Malatesta ${ }^{1}$

${ }^{1}$ Department of Neurosciences, Biomedicine and Movement, Anatomy and Histology Section, University of Verona; ${ }^{2}$ Department of Computer Science, Università di Verona; ${ }^{3}$ Center for Neuroscience and Cognitive Systems @UniTn, Istituto Italiano di Tecnologia, Rovereto; and ${ }^{4}$ Department of Biotechnology and Biosciences, University of Milano-Bicocca, Italy

E-mail: laura.calderan@univr.it

During last years, evidence has been provided on the involvement of obesity in the pathogenesis and aggravation of several lifethreatening diseases. In this view, we set up an innovative protocol to induce a nanoparticle-mediated lipolysis in vitro by combining light and electron microscopy, and biochemical approaches. Under appropriate administration conditions, 3T3-L1 mouse adipocytes proved to efficiently and safely internalize superparamagnetic iron oxide nanoparticles (SPIONs), which are able to produce heat when subjected to alternating magnetic field ${ }^{1-3}$. Thus, 3T3-LI adipocytes were submitted to SPIONs-mediated hyperthermia treatment (SMHT), with the aim of modulating their lipid content ${ }^{4,5}$. The treatment resulted in a significant delipidation persisting for at least $24 \mathrm{~h}$, in the absence of cell death, damage or dedifferentiation. Interestingly, some factors normally linked with lipolysis event or in lipid metabolism ${ }^{6}$ were not modulated upon SMHT, suggesting the involvement of a novel/alternative mechanism in the lipolysis observed. Notably, the same SMHT was able to induce delipidation also in primary cultures of human adipose-derived adult stem cells. The success of this new approach in vitro opens promising perspectives for the application of SMHT in different biomedical fields. Next steps could be the use of SPIONs in an animal model of obesity, to analyze the metabolic pathways activated by the SMHT, and the application of SMHT in an experimental model of cancer in obese mice to study the crosstalk between adipose and tumor tissues.

\section{References}

Lee JH, et al. Nat Nanotechnol 2011;6:418-22.

Guardi P, et al. ACS Nano 2012;6:3080-91.

Orlando T, et al. Contrast Media Mol Imaging 2016;11:139-45.

Bernabucci U, et al. J Mol Endocrinol 2009;42:139-47.

Sharifi S, et al. Sci Rep 2013;3:2173.

Brasaemle DL. J Lipid Res 2007;48:2547-59. 


\section{BIODERIVED POROUS SILICA HYBRID NANOVECTOR FOR THERANOSTIC APPLICATIONS}

R. Moretta ${ }^{1,2}$, M. Terracciano ${ }^{1}$, I. Rea ${ }^{1}$, P. Dardano ${ }^{1}$, L. De Stefano ${ }^{1}$

${ }^{1}$ Institute for Microelectronics and Microsystems; ${ }^{2}$ Department of Chemistry, University of Naples "Federico II", Naples, Italy

E-mail: luca.destefano@na.imm.cnr.it

Theranostic nanomedicine, which represents the development of integrated nanotherapeutic systems able to diagnose, deliver targeted therapy and monitor the response to the therapy, has led to a significant evolution in the research in the biomedical field. Innovative theranostic nanodevices could be designed by combining nanoparticles (NPs) with peculiar properties, able to deliver both diagnostic imaging agents and therapeutics at the same time. To this aim, several kinds of inorganic and organic nanoplatforms have been developed $^{1}$. Semiconductor quantum dots, magnetic and gold NPS are few examples of inorganic NPs proposed for fluorescent, magnetic and photoacoustic imaging, respectively 2,3 . Moreover, synthetic porous silica nanostructures were successfully used as carriers for drug delivery, despite their safety has not been demonstrated yet ${ }^{4}$. In order to develop biocompatible nanovectors, natural porous silica, obtained by diatoms, were recently exploited. Such inorganic nanoplatform is a cheap fossil compound, formed by fragments of diatom skeletons, called frustules ${ }^{5}$. The main constituent of diatomites is amorphous silica, approved as food grade by Food and Drug Administration (FDA). In vivo studies demonstrated that diatomite-derived NPs did not induce observable abnormalities in the organs of the mice nor tissue damage ${ }^{6}$. Herein, we explore the fabrication of a hybrid multifunctional nanodevice made of polyethylene glycol (PEG)-modified DNPs (PEG-DNPs) covered by gold NPs (AuNPs) for theranostic applications. Preliminary tests of uptake in HeLa cells, using AuNPs/PEG-DNPs labelled with Alexa 488 , demonstrated the perinuclear localization of the nanocomplexes.

\section{References}

1.Lim E-K, et al. Chem. Rev 2015;115:327-94.

2. Gao X, et al. Nat Biotechnol 2004;22:969-76.

3. Wang $S$, et al. Theranostics 2016;6:2394-413.

4 Park. JS, et al. Small 2011;7:2061-9.

5. Rea I, et al. Biochim Biophys Acta 2014;1840:3393-403.

6. Delalat B, et al. Nat Commun 2015;6:8791.

\section{PHYSICO-CHEMICAL CHARACTERIZATION OF DRUG NANOCARRIERS: A CRUCIAL STEP OR A WASTE OF TIME?}

S. Sennato ${ }^{1,2}$, A. Sarra ${ }^{2}$ C. Panella La Capria ${ }^{2}$, C. Bombelli³, E. Donati ${ }^{3}$, F. Bordi ${ }^{2}$

${ }^{1}$ Institute of Complex Systems (ISC), CNR, Rome; ${ }^{2}$ Department of Physics, La Sapienza University of Rome; and ${ }^{3}$ Institute for Biological Systems (ISB), CNR, Rome, Italy

\section{E-mail:simona.sennato@romal.infn.it}

Recently great effort has been devoted to the development of nanosized-carriers of different nature able to efficiently entrap biologically active compounds. Notably, materials with nanometric dimensions have distinct physicochemical properties in terms of size, surface properties, shape, composition, molecular weight, purity, stability, and solubility, which are crucial determinants of their physiological behaviour ${ }^{1}$. The key parameters in the physicochemical properties of a nano-formulation that are typically delineated in the literature are drug entrapment, stability, release kinetics and particle size and zeta potential. More, the so desired actual number of particles in formulations is of importance for comprehensive physicochemical characterization, pharmacodynamics and quality assurance. Currently there are no validated experimental methods for determining the particle number-concentration (Nc) of nanoparticle formulations. since theoretical calculation methods rely on several assumptions ${ }^{2}$ and other empirical counting methods suffer of different limitations ${ }^{3}$. Here we propose a new method for determining the number of particles based on high performance Laser Transmission Spectroscopy (LTS) ${ }^{4}$ which measures the transmittance of a laser beam through a suspension of particles as a function of the wavelength. The particle density distribution, as a function of their size, can be calculated through the Beer-Lambert law technique. Compared with traditional diffusion techniques (static and dynamic light scattering), LTS allows not only to study the size and shape of the particles in suspension but also to determine their absolute concentration Nc. In this study measurements on liposomal suspensions of HSPC:Chol and HSPC:DPPG prepared with extrusion techniques followed by sephadex purification have been performed by LTS and particle size distribution and Nc have been determined. Results obtained by LTS have been compared with those obtained by dynamic light scattering to shed light on some critical issue of this commonly used characterization technique.

\section{References}

1. Manaia E, et al. Int J Nanomedicine 2017;12:4991-5011.

2. Epstein $\mathrm{H}$, et al. Biomaterials 2006;27:651-9,

3. Sowerby S, et al. Sensors Actuators B 2007;123:325-30

4. Li F, et al. Appl Optics 2010;49:6602-11.

\section{NANOMATERIALS IN THE FRAMEWORK OF REACH \& CLP REGULATIONS}

M. Alessandrelli, R. Draisci

National Centre of Chemicals, Cosmetics and Consumer Protection (CNSC), Istituto Superiore di Sanità (ISS), Italy

E-mail: maria.alessandrelli@iss.it

Nanomaterials and nanotechnologies are a high-powered technicalscientific field and new challenges are emerging in the legislative framework regarding the specific evaluation and adequate management of potential risks related to nanomaterials. Currently no specific legislation solely devoted to nanomaterials is available but nanomaterials are covered by existing sector-specific legislations (cosmetics, biocides, food, medical devices) ${ }^{1}$. Nanomaterials are chemicals and as such they are included in the framework of REACH (EC Regulation No. 1907/2006)2 and CLP (EC Regulation No. 1272/2008) $)^{3}$, overarching regulations that apply to chemicals in any size, shape or physical state. CLP ${ }^{4}$ is based on intrinsic hazardous properties of the substances and mixtures: the nanospecific behaviour suggests that specific guidelines are required. CLP does not yet contain specific definitions or provisions for nanomaterials whereas for REACH, on 3 December 2018, the EU Commission adopted the Regulation (EU) 2018/18815 which introduces the nanoforms of substances and nanospecific prescriptions by modifying the annexes I, III, VI, VII, VIII, IX, X, XI and XII. The EU Commission, in cooperation with the European Chemical Agency (ECHA) and world organizations, such as GHS$O N U$ and $O E C D$, recognize the compelling need to modify existing regulations, adapting them to nanospecific perspective. Scientific developments have led to promote the systematic amendment review of OECD test guidelines and technical documents. To make sure of the significant and safe impact of nanomaterials placed on the market, it is important to integrate the high technical advantages by applying these materials with the scientific results of the risk evaluation in order to ensure regulatory oversight for nanomaterial and safety management of nanomaterials.

\section{References}

1. Mech A, et al. Nanotoxicology 2019;13:119-141

2. EU Regulation (EC) No. 1907/2006 concerning the Registration, Evaluation, Authorisation and Restriction of Chemicals (REACH).

3. EU Regulation (EC) No. 1272/2008 on classification, labelling and packaging of substances and mixtures, OJ L 353

4. Alessandrelli M, Polci ML. Annali ISS 2011;47:146-52.

5. Commission Regulation (EU) $2018 / 1881$ amending REACH as regards Annexes I, III, VI, VII, VIII, IX, X, XI, and XII to address nanoforms of substances OJ L 308. 